\title{
The involvement of protein kinase $C$ and actin filaments in cortical granule exocytosis in the rat
}

\author{
E Eliyahu, A Tsaadon, N Shtraizent and R Shalgi \\ Department of Cell and Developmental Biology, Sackler Faculty of Medicine, Tel Aviv University, Ramat Aviv, \\ Tel Aviv 69978, Israel
}

Correspondence should be addressed to R Shalgi; Email: shalgir@post.tau.ac.il

\begin{abstract}
Mammalian sperm-egg fusion results in cortical granule exocytosis (CGE) and resumption of meiosis. Studies of various exocytotic cells suggest that filamentous actin (F-actin) blocks exocytosis by excluding secretory vesicles from the plasma membrane. However, the exact function of these microfilaments, in mammalian egg CGE, is still elusive. In the present study we investigated the role of actin in the process of CGE, and the possible interaction between actin and protein kinase $C$ (PKC), by using coimmunoprecipitation, immunohistochemistry and confocal microscopy. We identified an interaction between actin and the PKC alpha isoenzyme in non-activated metaphase II (MII) eggs and in eggs activated by phorbol ester 12-O-tetradecanoyl phorbol-13-acetate (TPA). F-actin was evenly distributed throughout the egg's cytosol with a marked concentration at the cortex and at the plasma membrane. A decrease in the fluorescence signal of F-actin, which represents its depolymerization/ reorganization, was detected upon fertilization and upon parthenogenetic activation. Exposing the eggs to drugs that cause either polymerization or depolymerization of actin (jasplakinolide (JAS) and cytochalasin D (CD) respectively) did not induce or prevent CGE. However, CD, but not JAS, followed by a low dose of TPA doubled the percentage of eggs undergoing complete CGE, as compared with TPA alone. We further demonstrated that myristoylated alanin-rich C kinase substrate (MARCKS), a protein known to cross-link F-actin in other cell types, is expressed in rat eggs and is colocalized with actin. In view of our results, we suggest that the cytoskeletal cortex is not a mere physical barrier that blocks CGE, but rather a dynamic network that can be maneuvered towards allowing CGE by activated actin-associated proteins and/or by activated PKC.

Reproduction (2005) 129 161-170
\end{abstract}

\section{Introduction}

Sperm-egg fusion in mammals results in activation of the egg by triggering a series of biochemical events in a predetermined developmental program. A very early cellular event observed following sperm-egg fusion is an increase in intracellular calcium concentration $\left(\left[\mathrm{Ca}^{2+}\right]_{\mathrm{i}}\right.$ ) followed by oscillations. Other early events are cortical granule exocytosis (CGE), which leads to modification of the zona pellucida (ZP) and hence to the block of polyspermy and resumption of meiosis (Ben-Yosef \& Shalgi 1998, Swann \& Parrington 1999, Carroll 2001, Runft et al. 2002).

Many biological systems in which $\mathrm{Ca}^{2+}$ serves as a second messenger are also regulated by protein kinase $C$ (PKC) through its effect, either positive or negative, on the cellular signal transduction processes (Nishizuka 1988). Various studies have demonstrated expression and even possible involvement of PKC in the egg activation process (Gangeswaran \& Jones 1997, Johnson \& Capco 1997, Raz et al. 1998b, Pauken \& Capco 2000, Luria et al. 2000, Eliyahu et al. 2001). There is evidence that activation of
PKC induces CGE, but there is probably no direct linkage between PKC activation and the exit from metaphase II (MII; Jones 1998, Raz et al. 1998a). Studies performed in our laboratory revealed that conventional PKC (CPKC) isoenzymes were activated at fertilization and upon parthenogenetic activation either by 12-O-tetradecanoyl phorbol13-acetate (TPA) or by 1-oleoyl-2-acetylglycerol (OAG), but not by the calcium ionophore ionomycin (Eliyahu \& Shalgi 2002). Furthermore, we were able to inhibit CGE by a myristoylated PKC pseudosubstrate (myrPKC $\Psi)$, which is a specific PKC inhibitor, thus suggesting that exocytosis can be triggered independently either by $\left[\mathrm{Ca}^{2+}\right]_{i}$ rise or by PKC. In a recent study, it was demonstrated that cPKCs translocate to the egg plasma membrane following a pattern that is shaped by the amplitude, duration and frequency of $\mathrm{Ca}^{2+}$ transients (Halet et al. 2004).

PKC is known to be associated with a wide range of cytoskeletal components and/or to phosphorylate them (Inagaki et al. 1987). The actin cytoskeleton has long been proposed as the regulatory site of exocytosis. Evidence 
from several cell types suggests that a membrane-associated filamentous actin (F-actin) acts as a barrier to exocytosis by excluding secretory vesicles from the plasma membrane (Burgoyne et al. 1989, Vitale et al. 1991, Trifaro et al. 1992) and that its depolymerization is a primary step in the translocation of secretory vesicles to the plasma membrane (Lelkes et al. 1986, Sontag et al. 1988, Muallem et al. 1995). However, other studies have demonstrated polymerization, rather than depolymerization, of actin in some somatic cells undergoing exocytosis (Sha'afi \& Molski 1987, Apgar 1991). The presence of a subplasmalemal, actin-based cytoskeleton in eggs is well known (Spudich et al. 1988, Bonder et al. 1989, Roeder \& Gard 1994, Becker \& Hart 1996). The microfilaments of this cytoskeleton play a role in many dynamic processes during mammalian egg maturation and fertilization (Sutovsky et al. 1997, Connors et al. 1998, Terada et al. 2000). Processes such as sperm incorporation, CGE, spindle movement and polar body II (PBII) extrusion (Capco et al. 1992, Gallicano et al. 1992, 1995, Wang et al. 2000) are accompanied by reorganization of the actin cytoskeleton (Terada et al. 2000).

Various studies investigating the effect of actin-perturbing drugs on CGE have produced inconclusive results. CGE could be inhibited in the eggs of only some vertebrate species and only after treatment with some of the actin-perturbing drugs (Tahara et al. 1996, DiMaggio et al. 1997, Terada et al. 2000, McAvery et al. 2002), while other studies showed no inhibition of CGE (Boyle et al. 2001, Sun et al. 2001). Results regarding the effect of PKC activators on actin filaments are also ambiguous. Activation of PKC by TPA or by diacylglycerol (DAG) caused polymerization of actin, while PKC antagonist inhibited the formation of actin storage (Takashi 1997). However, in other studies, stimulation of PKC by phorbol esters was accompanied by a focal and transient disruption of the cortical F-actin network (Vitale et al. 1991, Tchakarov et al. 1998, Trifaro et al. 2002). These contradictory results reflect the lack of clarity regarding the role of PKC, actin and actin-associated proteins in the regulation of secretory events (Muallem et al. 1995).

Evidence from several cell types suggests that F-actin is associated with myristoylated alanin-rich $C$ kinase substrate (MARCKS; Rosen et al. 1990, Aderem 1992, Swierczynski \& Blackshear 1995, Rossi et al. 1999). MARCKS cross-links actin filaments and anchors the actin network to the plasma membrane (Rossi et al. 1999). In the present study we investigated the possible interaction between actin and PKC, both of which are known to participate in exocytosis. We examined the hypothesis that the cortical actin filaments serve as a barrier that excludes cortical granules (CGs) from the membrane of MII rat eggs. Changes in the distribution of actin filaments occur as a result of PKC activation during egg activation and enable CGE. Our results demonstrated that CGE cannot be triggered by a simple polymerization/depolymerization of actin filaments but rather PKC activation is required as well.

\section{Materials and Methods}

\section{Isolation of eggs}

MII-ovulated eggs

For induction of ovulation, 23- to 25-day-old immature Wistar-derived female rats, were injected with $10 \mathrm{IU}$ human chorionic gonadotropin (hCG; Sigma), 48-54 h after administration of $10 \mathrm{IU}$ pregnant mares serum gonadotropin (PMSG; Syncro-part, France). Rats were killed $14 \mathrm{~h}$ after the hCG administration. Cumulus-enclosed MII eggs were isolated from the oviducal ampullae into TH medium (Ben-Yosef et al. 1995), supplemented with $0.4 \%$ BSA (Sigma). Cumulus cells were removed by a brief exposure to $400 \mathrm{IU} / \mathrm{ml}$ hyaluronidase (Sigma) in $\mathrm{TH}$ medium.

\section{In vivo fertilized eggs}

PMSG-hCG-primed immature female rats were caged overnight with fertile males. Rats were killed $15 \mathrm{~h}$ after hCG administration. Egg isolation and cumulus cell removal were performed as described above for MII eggs. The eggs were classified according to the various stages of fertilization (Eliyahu \& Shalgi 2002): sperm binding (SB), fertilization cone (FC) and PBII stages (0-15, 15-60 and 60-180 min after sperm attachment respectively).

\section{Parthenogenetic activation}

MII-ovulated eggs were parthenogenetically activated by two different activators that are capable of inducing full CGE in rat eggs (Raz et al. 1998a, Eliyahu \& Shalgi 2002). The first activator was TPA $(30-100 \mathrm{ng} / \mathrm{ml}$; Sigma); a 5 -min incubation in the presence of TPA followed by an additional 5- or 10-min incubation in fresh medium lacking the activator. A stock solution of $1 \mathrm{mg} / \mathrm{ml}$ TPA was prepared in dimethylsulfoxide (DMSO) and stored at $-20^{\circ} \mathrm{C}$. The second activator was calcium ionophore $(2 \mu \mathrm{M}$; ionomycin 407950, Calbiochem, San Diego, CA, USA); a 3-min incubation in the presence of ionomycin followed by an additional 17-min incubation in fresh medium lacking the activator. A stock solution of $10 \mathrm{mM}$ ionomycin was prepared in DMSO and stored at $4{ }^{\circ} \mathrm{C}$.

\section{Actin polymerization and depolymerization}

MII eggs were incubated for $30 \mathrm{~min}$ with the actin-depolymerizing drug cytochalasin D (CD; $10 \mu \mathrm{g} / \mathrm{ml}$, Sigma), or with the polymerizing drug jasplakinolide/jaspamide (JAS; $1 \times 10^{-7} \mathrm{nM}$, Molecular Probes, Eugene, OR, USA).

\section{Antibodies and staining agents}

Primary antibodies: anti-actin rabbit polyclonal $\lg G$ (A-5060, Sigma); anti-cPKC alpha mouse monoclonal IgG (P-16520, Transduction Laboratories, Lexington, KY, USA); anti-MARKCS goat polyclonal IgG (sc-6454, Santa Cruz, CA, USA). Peroxidase-conjugated secondary antibodies: 
goat anti-rabbit IgG (sc-2004, Santa Cruz); goat antimouse IgG (sc-2005, Santa Cruz); donkey anti-goat IgG (sc-2020, Santa Cruz). Cy-conjugated secondary antibody: donkey anti-goat IgG (Jackson Immunoresearch Laboratories, PA, USA). Staining agents: Texas Red phalloidin (T-7471, Molecular Probes); Biotinylated lens culinaris agglutinin (LCA)-biotin (B-1045, Vector, Burlingame, CA, USA); Texas Red streptavidin (SA-5006; Vector).

\section{Immunoprecipitation and immunoblotting}

An antibody against anti-actin was chemically coupled to protein A sepharose in order to prepare an immobilized antibody affinity reagent for immunoprecipitation (IP buffer), according to Talmor et al. (1998). Batches of 1000 eggs, that either had or had not been subjected to activating agents, were lysed in $100 \mu \mathrm{I}$ IP buffer and kept at $-70^{\circ} \mathrm{C}$. Upon thawing, the egg lysates were incubated overnight at $4{ }^{\circ} \mathrm{C}$ with $10 \mu \mathrm{l}$ immobilized antibody (25\% suspension) and then washed by centrifugation with IP buffer and separated by $10 \%$ SDS-PAGE under non-reducing conditions. Proteins were transferred onto a nitrocellulose membrane (Amersham) using a wet blotting apparatus (Hoeffer, San Francisco, CA, USA). For immunoblot analysis, blots were blocked with Tris-buffered saline with $5 \%$ dry milk and incubated in the presence of various specific primary antibodies. Bound antibodies were recognized by secondary antibodies conjugated to horseradish peroxidase. Detection was performed by an ECL detection system (Pierce, Rockford, IL, USA). Approximate molecular masses were determined by comparison with the migration of prestained protein standards (Amersham).

\section{Immunofluorescence staining and laser-scanning confocal microscopy}

Eggs at various developmental stages were fixed for 10 min at room temperature in Dulbecco's phosphate-buffered saline (DPBS) with 3\% paraformaldehyde and $0.01 \%$ glutaraldehyde. Eggs were then washed in a solution of $3 \%$ foetal calf serum (FCS; Biological Industries, Beit-Haemek, Israel) in DPBS (DPBS/FCS). ZPs were removed post-fixation by $0.25 \%$ pronase (Sigma) in DPBS/FCS. The ZP-free eggs were washed in DPBS/FCS. For detection of CGE, fixed eggs were labeled by LCA and Texas Red streptavidin (Eliyahu \& Shalgi 2002).

For labeling egg proteins, the plasma membrane of ZPfree eggs was permeabilized during 10-min incubation in $0.05 \%$ NP-40 in DPBS/FCS solution followed by several rinses in $0.005 \% \mathrm{NP}-40$ in DPBS/FCS.

For actin labeling eggs were incubated for $2 \mathrm{~h}$ in the presence of Texas Red phalloidin $(1.1 \mathrm{nM})$ or with antiMARCKS $(1: 100)$ followed by an incubation in the presence of a fluorescence-labeled Cy secondary antibody (1:300). Chromatin labeling and the fertilization stage of the eggs were assessed by incubating the eggs for $10 \mathrm{~min}$ in the presence of $1 \mu \mathrm{g} / \mathrm{ml}$ Hoechst (Sigma). The progression of eggs past the MII stage was analyzed by moni- toring the separation of the chromosomal dyads. The various stages of fertilization were determined by following the sperm and egg chromatin. The labeling of CG exudates, actin, MARCKS and DNA was visualized and photographed by a Zeiss confocal laser-scanning microscope (CLSM; Oberkochen, Germany). The Zeiss LSM 410 is equipped with a $25 \mathrm{~mW}$ krypton-argon laser, a $10 \mathrm{~mW}$ helium-neon laser (488, 543 and 633 maximum lines) and an u.v. laser (Coherent Inc. Laser Group, Santa Clara, CA, USA). A $\times 40$ numerical aperture/1.2 planapochromat water immersion lens (Axiovert $135 \mathrm{M}$, Zeiss) was used for all imaging. Eggs were scanned using the CLSM through the $Z$-axis to perform a section at the equatorial plane of the egg. The stain intensity was measured using the correct mean density values obtained by the LSM software. The fluorescence intensity was expressed relative to values obtained for MII eggs, which were set to 1.0 (arbitrary units).

\section{Results}

In the present study we investigated the possible interaction between actin and PKC at the pathway leading to CGE during the process of egg activation.

\section{Expression of actin in the egg}

We used Western blot analysis to evaluate the total amount of actin in non-activated MII eggs and in TPA-activated eggs. Egg proteins, before or after parthenogenetic activation of the eggs by TPA, were separated on SDSPAGE. As shown in Fig. 1, the amount of actin, a $46 \mathrm{kDa}$ protein, was similar in both non-activated MII eggs and in TPA-activated eggs.

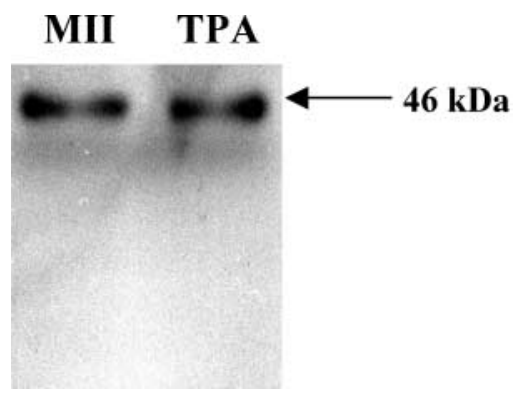

Figure 1 Detection of total actin (F-and G-actin) in non-activated MII and TPA-activated eggs. Eggs, before or after parthenogenetic activation by $50 \mathrm{ng} / \mathrm{ml}$ TPA, were pooled, lysed and the proteins separated by SDS-PAGE (200 eggs per lane). The proteins were immunoblotted with anti-actin rabbit polyclonal IgG (1:250). Peroxidase-conjugated goat anti-rabbit IgG (1:5000) secondary antibodies were detected by an ECL detection system. A single band was detected at the appropriate molecular mass for actin (46 kDa), as calculated from the migration of protein standards with known molecular masses. At least three independent experiments were performed. 


\section{Association between actin and PKC alpha during egg activation by TPA}

Coimmunoprecipitation assays were performed to detect the possible interaction between actin and PKC. Lysates of eggs, before or after parthenogenetic activation by TPA, were immunoprecipitated with anti-actin as described in Materials and Methods. The nitrocellulose membrane was incubated in the presence of anti-PKC alpha monoclonal antibody. Our results show an interaction between PKC alpha and actin in non-activated MII eggs and in TPA-activated eggs (Fig. 2). As expected, a single $84 \mathrm{kDa}$ PKC alpha band was detected in non-activated MII eggs whereas TPA-activated eggs exhibited two bands, at 84 and $88 \mathrm{kDa}$, that may represent the unphosphorylated and the phosphorylated forms of PKC alpha respectively. Several treatments were used as control groups: IP buffer containing only the immune complex; incubation of the nitrocellulose membrane with any $\lg \mathrm{G}$ antibody (polyclonal anti-Src antibody); incubation of the egg lysate with any antibody (polyclonal anti-Src antibody) conjugated to protein A sepharose. None of the control groups exhibited either an $84 \mathrm{kDa}$ or an $88 \mathrm{kDa}$ band on the nitrocellulose membrane (data not shown).

\section{Distribution of F-actin during egg activation}

The intracellular distribution and organization of F-actin in fertilized or in parthenogenetically activated eggs was examined by immunofluorescence confocal microscopy. F-actin was evenly distributed throughout the cytosol of MIl-arrested eggs (Fig. 3A and D) with a marked concentration at the cortex and at the plasma membrane, especially above the metaphase plate (Fig. 4A and E). $A$ decrease in the fluorescence signal of F-actin was detected upon fertilization, at the SB stage, as compared with MII eggs (Fig. 3B and E), the decrease was more pronounced at the FC/PBII stages (Fig. 3C and F). In TPA-activated eggs, we observed a time-dependent decrease of the fluorescence signal (Fig. 4F-H), as compared with untreated MII eggs (Fig. 4E). As expected, eggs treated with TPA remained at the MII stage (Fig. 4B-D).

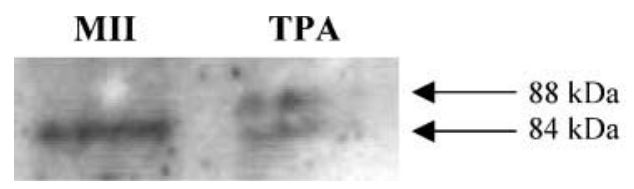

Figure 2 Association of $\mathrm{CPKC}$ alpha with actin upon egg activation by TPA. Eggs (1000 per lane), before (MII) or after (TPA) parthenogenetic activation by $50 \mathrm{ng} / \mathrm{ml}$ TPA, were lysed and immunoprecipitated with anti-actin rabbit polyclonal IgG (IP = actin). The proteins were immunoblotted with anti-cPKC alpha monoclonal IgG (1:500). Peroxidaseconjugated goat anti-mouse IgG (1:500) secondary antibody was detected by an ECL detection system. Two bands were detected at 84 and $88 \mathrm{kDa}$, corresponding to CPKC alpha and phosphorylated CPKC alpha respectively (arrows), as calculated from the migration of protein standards with known moleclar masses. At least three independent experiments were performed.
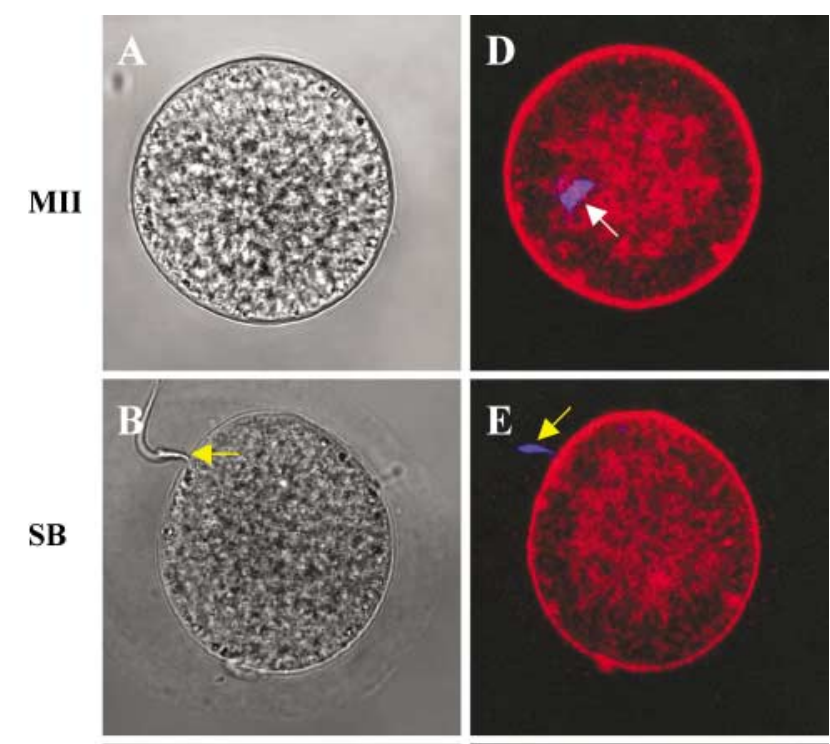

\section{FC}
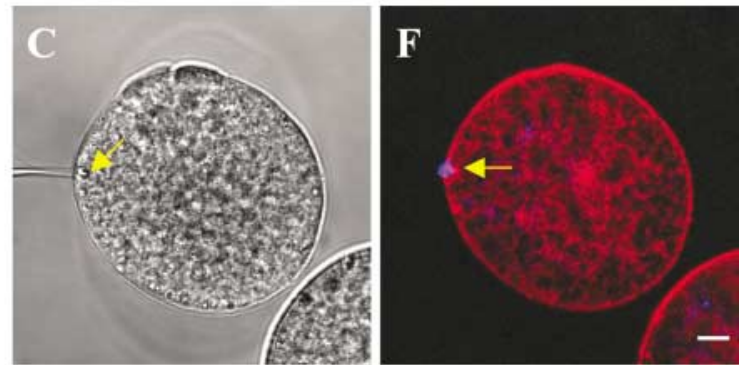

Figure 3 Distribution of F-actin at various stages of in vivo fertilization. Fluorescence intensity was calculated as described in Materials and Methods. The values were expressed relative to those obtained for MII eggs, which were set to 1.0 (arbitrary units). Fertilized eggs were fixed and labeled with Texas Red phalloidin (1.1 nM; F-actin labeling) and with Hoechst $(1 \mu \mathrm{g} / \mathrm{ml}$; DNA labeling) and imaged using a laser-scanning confocal microscope. (A and D) Unfertilized egg (MII, relative fluorescence $=1.0)$. (B and $E$ ) Egg at the sperm binding $(\mathrm{SB})$ stage (relative fluorescence $=0.68)$. $(\mathrm{C}$ and $\mathrm{F}$ ) Egg at the fertilization cone $(F C)$ stage (relative fluorescence $=0.68$ ). Light microscopy $(\mathrm{A}-\mathrm{C})$; actin and chromosomes $(\mathrm{D}-\mathrm{F})$; sperm DNA (yellow arrow); egg DNA (white arrow). At least three independent experiments (three to four eggs for each group in each experimental day) were performed. Each image was taken at the equatorial plane of the egg. Scale bar, $10 \mu \mathrm{m}$.

The temporal decrease observed in the fluorescence intensity of TPA-activated eggs might reflect the depolymerization/reorganization of F-actin.

\section{Effect of actin polymerizing/depolymerizing drugs on the distribution of F-actin and on CGE}

To evaluate the role of F-actin in CGE, we incubated eggs in the presence of drugs that induce polymerization or depolymerization of actin (JAS or CD respectively). The distribution of F-actin, before and after incubation in the presence of either drug, was followed (Fig. 5). CD caused aggregation of F-actin within the cytoplasm and a decreased fluorescence intensity, reflecting depolymerization of actin (Fig. 5E). JAS, on the other hand, caused an 

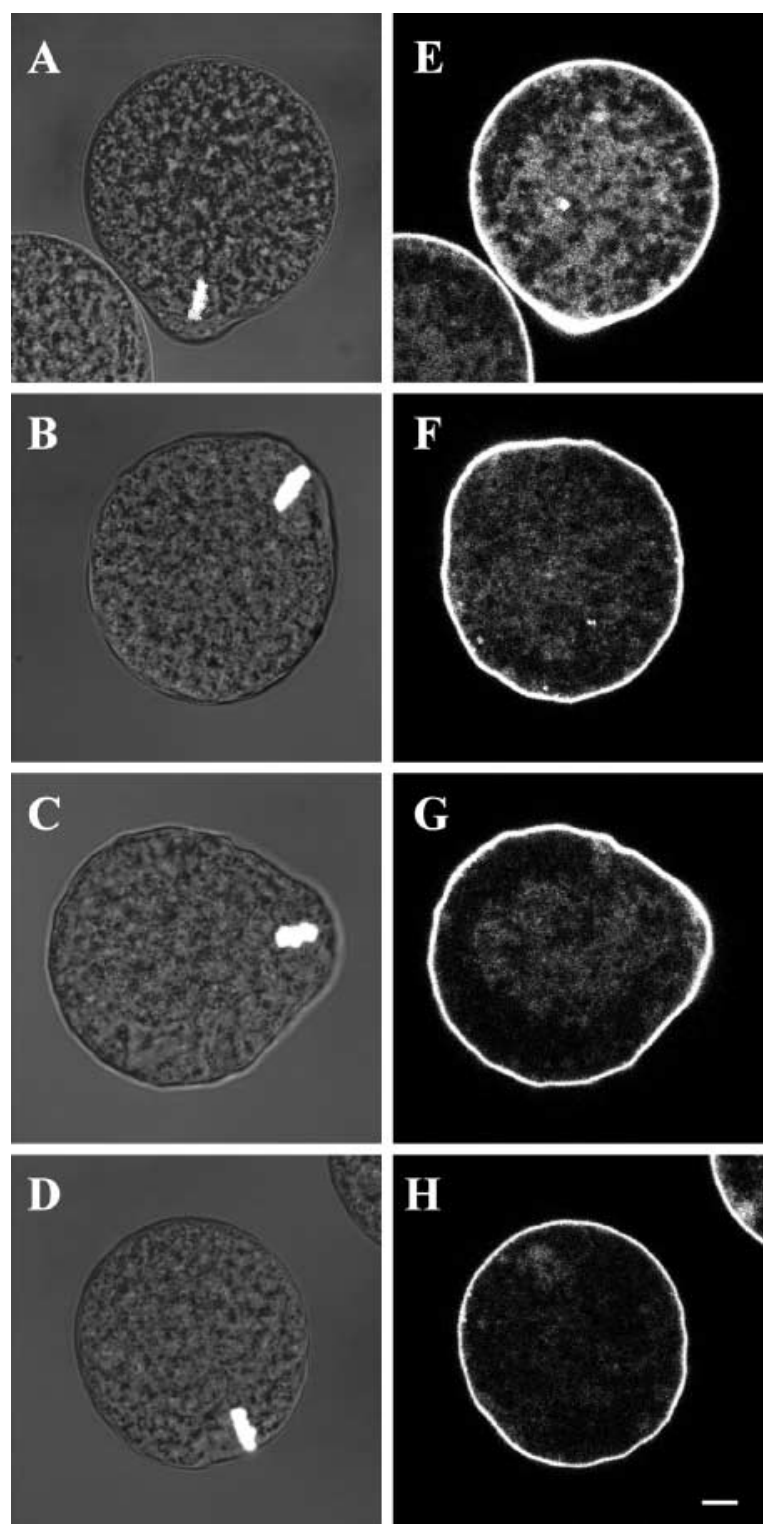

Figure 4 Distribution of F-actin during parthenogenetic activation by TPA. Fluorescence intensity was calculated as described in Materials and Methods. The values were expressed relative to those obtained for MII eggs, which were set to 1.0 (arbitrary units). Eggs were fixed at the MII stage $(A$ and $E$; relative fluorescence $=1.0)$ or after incubation for 5 ( $\mathrm{B}$ and $\mathrm{F}$; relative fluorescence $=0.40), 10(\mathrm{C}$ and $\mathrm{G}$; relative fluorescence $=0.43)$ or $15(\mathrm{D}$ and $\mathrm{H}$; relative

fluorescence $=0.20) \mathrm{min}$ in the presence of $100 \mathrm{ng} / \mathrm{ml}$ TPA. Eggs were labeled with Texas Red phalloidin (1.1 nM; F-actin labeling) and with Hoechst $(1 \mu \mathrm{g} / \mathrm{ml}$; DNA labeling). Localization of the actin was imaged using a laser-scanning confocal microscope. Light microscopy and chromosomes (A-D); actin $(E-H)$. At least three independent experiments (three to four eggs for each group in each experimental day) were performed. Each image was taken at the equatorial plane of the egg. Scale bar, $10 \mu \mathrm{m}$.

increase in the fluorescence intensity of F-actin throughout the egg, reflecting polymerization of F-actin (Fig. 5F). Eggs treated with either $\mathrm{CD}$ or JAS did not progress beyond the Mll stage (Fig. 5A-C).
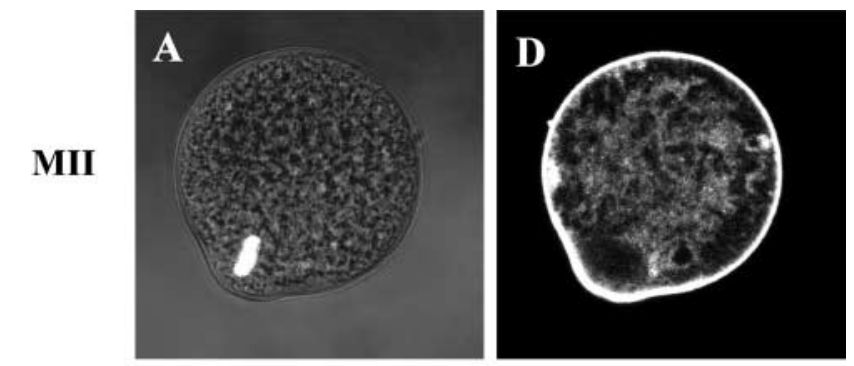

CD
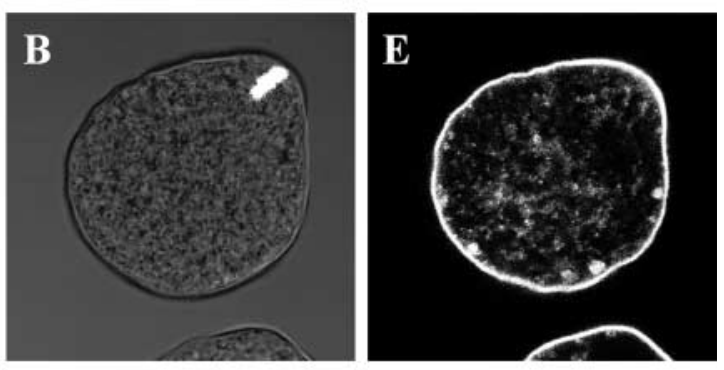

JAS
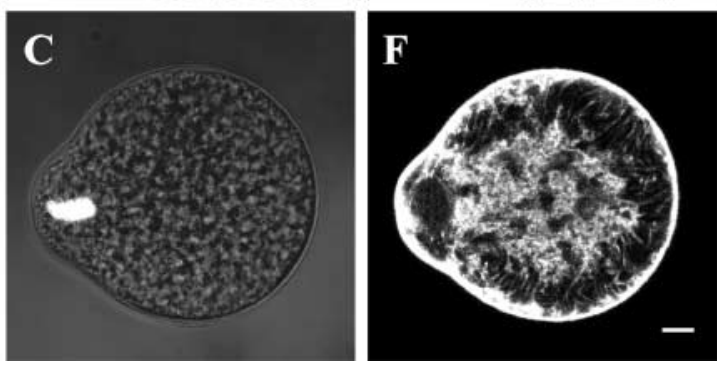

Figure 5 Effect of actin-polymerizing/-depolymerizing drugs on the distribution of F-actin. Eggs, before or after 30-min incubation in the presence of either $10 \mu \mathrm{g} / \mathrm{ml} \mathrm{CD}$ or $10^{-7} \mathrm{mM}$ JAS were fixed and labeled with Texas Red phalloidin (1.1 nM; F-actin labeling) and with Hoechst $(1 \mu \mathrm{g} / \mathrm{ml}$; DNA labeling). Non-treated eggs were fixed at the MIl stage ( $A$ and $D)$. Treated eggs were fixed after exposure to $C D$ ( $\mathrm{B}$ and $\mathrm{E}$ ) or to JAS (C and F). Localization of actin was imaged using a laser-scanning confocal microscope. Light microscopy and chromosomes (A-C); actin (D-F). At least three independent experiments (three to four eggs for each group in each experimental day) were performed. Each image was taken at the equatorial plane of the egg. Scale bar, $10 \mu \mathrm{m}$.

The correlation between PKC activation, F-actin reorganization and CGE was examined by monitoring the capability of eggs, exposed to CD or to JAS, to undergo CGE while PKC was activated by TPA (Fig. 6). MII eggs were incubated in the presence of either CD or JAS and then activated by $30 \mathrm{ng} / \mathrm{ml} \mathrm{TPA}$ in the presence of either drug. Untreated MII eggs served as a negative control for CGE (Fig. 6A), whereas, eggs exposed only to TPA served as a positive control (Fig. 6D). MIl eggs, as well as eggs exposed to either JAS or CD, did not undergo CGE (Fig. $6 \mathrm{~A}-\mathrm{C})$, nor did they overcome the second metaphase arrest (not shown). As expected, eggs treated with $30 \mathrm{ng} / \mathrm{ml}$ TPA, a concentration that triggers only partial CGE (Raz et al. 1998a), exhibited moderate fluorescence intensity (Fig. 6D). Addition of CD, but not of JAS, caused enhancement of the CGE fluorescence intensity, indicating that the eggs had undergone complete CGE JAS, Fig. 6E; CD, 

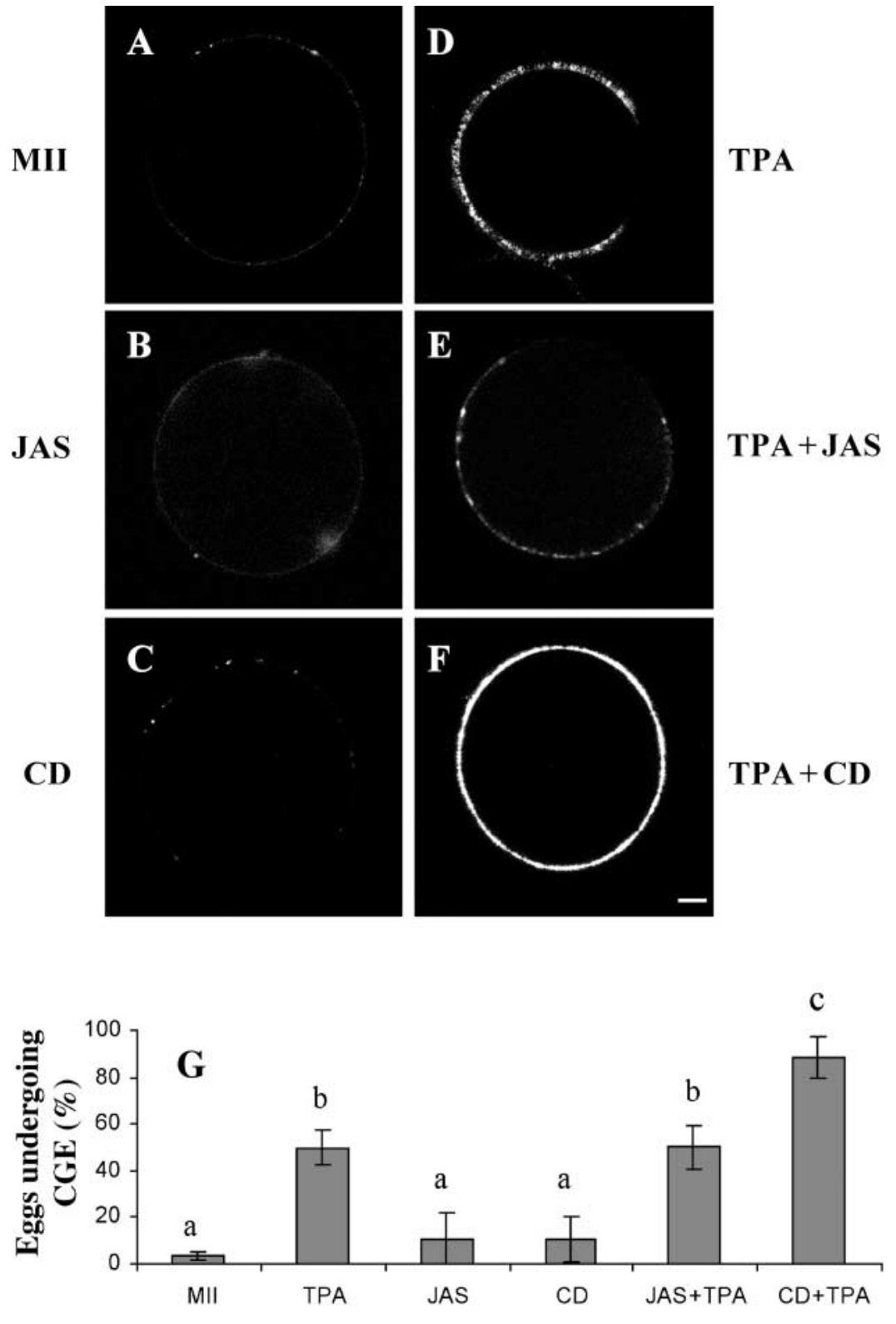

Treatment
TPA

TPA + JAS

Figure 6 Effect of actin-polymerizing/-depolymerizing drugs on CGE. (A-F) Immunofluorescence confocal microscopy. Eggs, before or after activation by $30 \mathrm{ng} / \mathrm{ml} \mathrm{TPA}$ in the presence of $10^{-7} \mathrm{mM} \mathrm{JAS}$ or $10 \mu \mathrm{g} / \mathrm{ml} \mathrm{CD}$, were fixed and labeled by LCAbiotin (1:100) followed by Texas Red-sterpavidin (1:1000) for CGE detection. Eggs were examined at the MIl stage (A); after 30-min incubation in the presence of JAS (B) or CD (C); after 5-min activation by TPA, followed by $25 \mathrm{~min}$ in fresh TH medium (D); or after 30-min incubation in the presence of JAS or CD followed by 5-min activation by TPA in the presence of JAS/CD (E and F respectively). The occurrence of the CGE process was imaged using a laser-scanning confocal microscope. At least three independent experiments (three to four eggs for each group in each experimental day) were performed. Each image was taken at the equatorial plane of the egg. Scale bar, $10 \mu \mathrm{m}$. (G) The percentage of eggs undergoing complete CGE under the various treatments. The intensity of the labeling was measured using the corrected mean density values obtained by the LSM image software. The $Y$-axis represents the percentage of eggs that underwent complete CGE. Values are means \pm S.E.M. TPA in the presence of $\mathrm{CD}$ induced significantly higher rates of CGE than TPA alone $(P<0.005$, two-way ANOVA test). Different letters represent significant differences.
Fig. 6F). There were no significant differences in the ability of eggs to undergo complete CGE between MII-arrested eggs and eggs treated with CD or JAS alone (Fig. 6G, $P>0.05$, two-way ANOVA test). However, exposing eggs to $\mathrm{CD}$ followed by exposure to $30 \mathrm{ng} / \mathrm{ml}$ TPA, doubled the percentage of eggs undergoing complete CGE as compared with eggs incubated with TPA alone (88.6 and $46.4 \%$ respectively, $P<0.005$, two-way ANOVA test).

\section{Expression of MARCKS in non-activated MII eggs and in activated eggs}

In various cell types, MARCKS, a myristoylated protein, cross-links F-actin and anchors the actin network to the plasma membrane. An important step towards understanding the role of MARCKS in the process of egg activation was the study of its expression, localization and colocalization with actin. Eggs, before or after parthenogenetic activation, were pooled, lysed and their proteins immunoblotted. As seen in Fig. 7, the egg lysates exhibited a protein band with an apparent molecular mass of $80 \mathrm{kDa}$, which is consistent with the expected molecular mass for MARCKS protein (Wu et al. 1982, Aderem 1992). The protein band of non-activated MII eggs was much stronger than that of TPA- or of ionomycin-activated eggs (Fig. 7). Control eggs were incubated in the presence of antiMARCKS primary antibody that had previously been exposed for $1 \mathrm{~h}$ to a specific MARCKS peptide $(2 \mu \mathrm{g} / \mathrm{ml})$. No band was observed in this lane (data not shown). 


\section{Iono TPA}

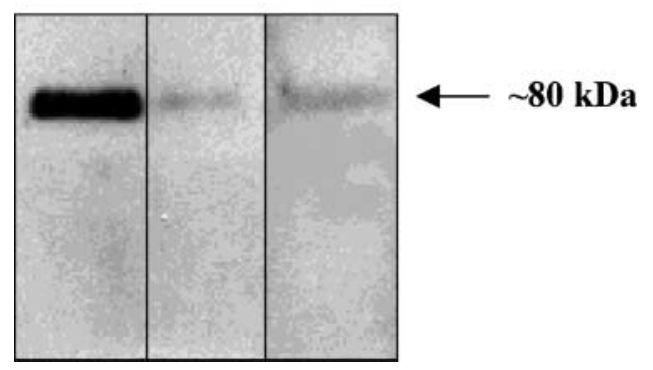

Figure 7 Expression of MARCKS in MII eggs and in eggs activated by TPA or by ionomycin. Eggs, before or after parthenogenetic activation by TPA $(50 \mathrm{ng} / \mathrm{ml})$ or by ionomycin $(2 \mu \mathrm{M})$, were pooled, lysed and the proteins separated by SDS-PAGE (400 eggs per lane). The proteins were immunoblotted with anti-MARKCS goat polyclonal $\lg \mathrm{G}(1: 200)$. Peroxidase-conjugated donkey anti-goat IgG secondary antibody was used (1:5000) followed by an ECL detection system. The arrow points to MARCKS at $\sim 80 \mathrm{kDa}$ as calculated from the migration of protein standards with known molecular masses. At least three independent experiments were performed.

To examine a possible interaction between MARCKS and actin, we double-labeled eggs for the two proteins and examined their colocalization by immunofluorescence confocal microscopy. As already demonstrated, F-actin was homogenously distributed throughout the cytosol of MII eggs with a marked localization at the membrane and cortex (Fig. 8C). MARCKS was highly concentrated at the membrane of MII eggs (Fig. 8B). A distinct colocalization between MARCKS and actin was noted at the plasma membrane (Fig. 8D). Our results demonstrate, for the first time, expression and localization of MARCKS protein and its colocalization with actin in the rat egg.

\section{Discussion}

The initial signal of egg activation, shortly after spermegg interaction, is an increase in $\left[\mathrm{Ca}^{2+}\right]_{i}$. PKC is also an active participant in some aspects of egg activation, as it is in other cell types (Kline \& Kline 1992, Ducibella et al. 1993, Raz \& Shalgi 1998, Eliyahu et al. 2002). The expression of various PKC isoenzymes and their capability to be activated in eggs have already been demonstrated (Gangeswaran \& Jones 1997, Johnson \& Capco 1997, Raz et al. 1998b, Luria et al. 2000, Pauken \& Capco 2000, Halet et al. 2004). In a recent study we showed that CGE can be triggered either by a $\left[\mathrm{Ca}^{2+}\right]_{i}$ rise or by PKC (Eliyahu \& Shalgi 2002). Since microfilaments play a role in many dynamic events during mammalian egg maturation and fertilization, and since PKC activation causes reorganization of actin leading to exocytosis in somatic cells (Lelkes et al. 1986, Sontag et al. 1988, Capco et al. 1992, Gallicano et al. 1992, Muallem et al. 1995, Takashi 1997, Wang et al. 2000), we chose to study the involvement of both actin and PKC in CGE. In the current work, we examined the affect of TPA, as a parthenogenetic activator, on the amount and distribution of total actin within the egg and on its interaction with PKC alpha.
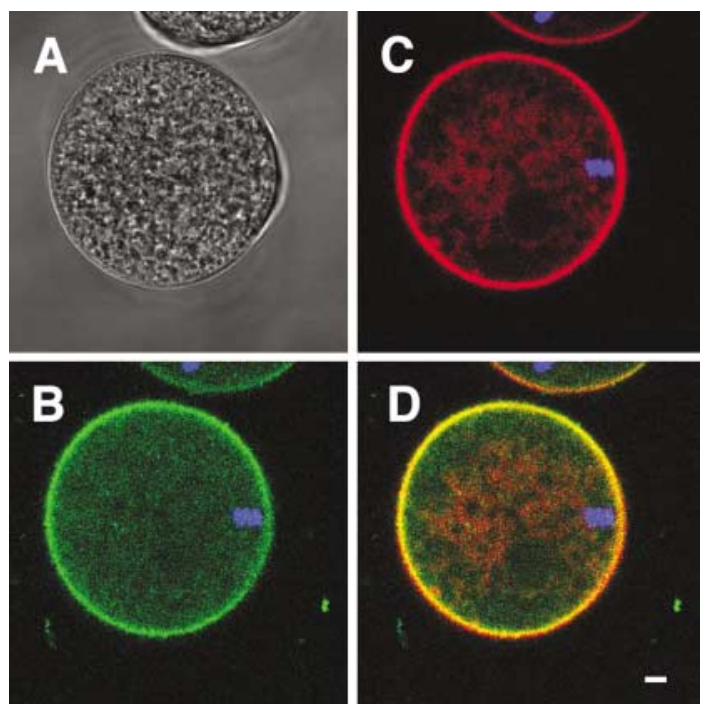

Figure 8 Immunofluorescence colocalization of MARCKS and Factin. Eggs were fixed at the MII stage and labeled with anti-MARKCS goat polyclonal IgG (1:50; MARCKS labeling; green), Texas Red phalloidin (1.1 nM; actin labeling; red) and Hoechst $(1 \mu \mathrm{g} / \mathrm{ml}$; DNA labeling; blue). Control eggs were labeled with the primary antibody, which had been previously incubated for $1 \mathrm{~h}$ with $2 \mu \mathrm{g} / \mathrm{ml}$ of the specific MARCKS peptide. Localization of the antibodies was imaged using a secondary donkey anti-goat IgG Cy antibody (1:300) and a laser-scanning confocal microscope. Light microscopy (A); MARCKS (B); F-actin (C); colocalization between the MARCKS and F-actin (D; yellow). At least three independent experiments (three to four eggs for each group in each experimental day) were performed. Each image was taken at the equatorial plane of the egg. Scale bar, $10 \mu \mathrm{m}$.

The amount of actin in both non-activated MII eggs and in TPA-activated eggs was similar. This finding is not surprising as most cells have large actin storage ready for an immediate response to intracellular events (Welch et al. 1997). A coimmunoprecipitation assay was used to identify a possible, direct or indirect, interaction between PKC and actin. A band of PKC alpha, an isoenzyme known to participate in exocytosis in other cell types (Benussi et al. 1998) was detected in lysates of non-activated MII eggs. Lysates of activated eggs exhibited two bands of PKC alpha, which probably represent the unphosphorylated and the phosphorylated forms of the isoenzyme as described by Bornancin \& Parker (1997). cPKC isoenzymes are known to undergo autophosphorylation during activation. The interaction observed between actin and the phosphorylated and unphosphorylated forms of PKC implies an involvement of both proteins in the egg activation process.

Non-activated MII eggs demonstrated a homogenous distribution of F-actin throughout the cytosol as well as a specific localization at the egg membrane, mainly above the meiotic spindle. Activation of the eggs by sperm or by TPA caused depolymerization/reorganization of the F-actin, as manifested by a decrease in the intensity of the fluorescence signal. The most significant changes observed took place in the cytosol and not at the plasma 
membrane. We attribute it to the strong actin signal detected at the plasma membrane of non-activated MII eggs, which can mask minor fluorescence changes. Another possibility is that the actin- binding proteins that anchor the F-actin to the plasma membrane render the membranous actin more stable than the cytosolic actin. However, since the total amount of actin appeared similar in Western blots of both activated and non-activated eggs, we can safely assume that there is a change in the ratio of G-actin to F-actin.

TPA induced polymerization of actin in Tubifex eggs while PKC antagonists inhibited the polymerization (Takashi 1997). Depolymerization of actin by CD enhances CGE in zebrafish eggs, while polymerization of actin by phalloidin inhibits CGE (Becker \& Hart 1999). They conclude that F-actin acts as a mechanical barrier to exocytosis by excluding CG vesicles from the plasma membrane and that this barrier has to be removed prior to membrane fusion. To study the affect of PKC activation on actin reorganization eggs were treated with actin-polymerizing and -depolymerizing drugs. As expected, CD caused depolymerization of actin while JAS caused polymerization. In an attempt to follow the interplay between F-actin reorganization, PKC activation and CGE, eggs were treated with either CD or JAS with or without a further exposure to TPA. Although CD or JAS alone did not induce CGE, CD but not JAS increased the intensity of the CGE fluorescence signal caused by TPA. The mechanism by which CGs are anchored to the egg cortex is unclear. The observation that $\mathrm{CD}$ did not cause spontaneous CGE indicates that the reorganization of actin is not adequate to allow CGE, thus implying that the egg cytoskeletal cortex is not a simple barrier that blocks constitutive exocytosis, as suggested for other cells. A more likely possibility is that actin-associated proteins and/or PKC need to be activated in order to cause CGE. We suggest that these proteins are activated in response to parthenogenetic activation by TPA, and they, in turn, could cause the remodeling of the cortical microfilamentous actin that is ultimately required for CGE.

Evidence from several cell types suggests that MARCKS cross-links F-actin and anchors the actin network to the plasma membrane (Rosen et al. 1990, Aderem 1992, Swierczynski \& Blackshear 1995, Rossi et al. 1999). An important initial step towards understanding the role of MARCKS during egg activation was the study of its expression, localization and colocalization with actin. We have shown that MARCKS is present in abundance in MII eggs but that levels decrease upon egg activation, as depicted by the decreased band intensity in Western blot analysis. We should bear in mind that the decrease in band intensity could also reflect a decrease in the affinity of the antibody to the protein, caused by phosphorylation of MARCKS. Immunohistochemistry and confocal scanning microscopy enabled us to demonstrate MARCKS localization at the plasma membrane of non-activated MII eggs and its colocalization with F-actin. In view of the aforementioned data, we assume that MARCKS is associated with F-actin and we suggest the hypothesis that, for CGE to occur, PKC has to phosphorylate an actin-bound protein such as MARCKS.

\section{Acknowledgements}

We thank Dr Leonid Mittelman for his excellent technical assistance at the confocal microscope and Ruth Kaplan-Kraicer for technical help and advice. This work was partially supported by a grant from the Ministry of Health to R S.

\section{References}

Aderem A 1992 The MARCKS brothers: a family of protein kinase C substrates. Cell $71713-716$.

Apgar JR 1991 Regulation of the antigen-induced F-actin response in rat basophilic leukemia cells by protein kinase C. Journal of Cell Biology 112 1157-1163.

Becker KA \& Hart NH 1996 The cortical actin cytoskeleton of unactivated zebrafish eggs: spatial organization and distribution of filamentous actin, nonfilamentous actin, and myosin. Molecular Reproduction and Development 43 536-547.

Becker KA \& Hart NH 1999 Reorganization of filamentous actin and myosin-II in zebrafish eggs correlates temporally and spatially with cortical granule exocytosis. Journal of Cell Science $\mathbf{1 1 2}$ 97-110.

Benussi L, Govoni S, Gasparini L, Binetti G, Trabucchi M, Bianchetti A \& Racchu M 1998 Specific role for protein kinase C alpha in the constitutive and regulated secretion of amyloid precursor protein in human skin fibroblasts. Neuroscience Letters 240 97-101.

Ben-Yosef D \& Shalgi R 1998 Early ionic events in activation of the mammalian egg. Reviews of Reproduction 3 96-103.

Ben-Yosef D, Oron Y \& Shalgi R 1995 Low temperature and fertilization-induced $\mathrm{Ca}^{2+}$ changes in rat eggs. Molecular Reproduction and Development 42 122-129.

Bonder EM, Fishkind DJ, Cotran NM \& Begg DA 1989 The cortical actin-membrane cytoskeleton of unfertilized sea urchin eggs. Analysis of the spatial organization and relationship of filamentous actin, nonfilamentous actin, and egg spectrin. Developmental Biology 134 327-341.

Bornancin F \& Parker PT 1997 Phosphorylation of protein kinase C- $\alpha$ on serine 657 controls the accumulation of active enzyme and contributes to its phosphatase-resistant state. Journal of Biological Chemistry 272 3544-3549.

Boyle JA, Chen H \& Bamburg JR 2001 Sperm incorporation in Xenopus laevis: characterization of morphological events and the role of microfilaments. Zygote 9 167-181.

Burgoyne RD, Cheek TR \& O'Sullivan AJ 1989 The control of cytoskeletal actin and exocytosis in intact and permeabilized adrenal chromaffin cells: role of calcium and protein kinase C. Cellular Signalling $1323-334$.

Capco DG, Tutnick JM \& Bement WM 1992 The role of protein kinase $\mathrm{C}$ in reorganization of the cortical cytoskeleton during the transition from oocyte to fertilization-competent egg. Journal of Experimental Zoology 264 395-405.

Carroll J 2001 The initiation and regulation of Ca2 + signaling at fertilization in mammals. Seminars in Cell and Developmental Biology 12 37-43.

Connors SA, Kanatsu-Shinohara M, Schultz RM \& Kopf GS 1998 Involvement of cytoskeleton in movement of cortical granules during oocyte maturation, and cortical granules anchoring in mouse eggs. Developmental Biology 200 103-115.

DiMaggio AJ Jr, Lonergan TA \& Stewart-Savage J 1997 Cortical granule exocytosis in hamster eggs requires microfilaments. Molecular Reproduction and Development 47 334-340. 
Ducibella T, Kurasawa S, Duffy P, Kopf GS \& Schults RM 1993 Regulation of the polyspermy block in the mouse egg: maturation-dependent differences in cortical granule exocytosis and zona pellucida modifications induced by inositol 1,4,5-trisphosphate and an activator of protein kinase C. Biology of Reproduction 48 1251-1257.

Eliyahu E \& Shalgi R 2002 A role for protein kinase C during rat egg activation. Biology of Reproduction 67 189-195.

Eliyahu E, Kaplan-Kraicer R \& Shalgi R 2001 PKC in egg and embryos. Frontiers in Bioscience $\mathbf{6}$ 785-791.

Eliyahu E, Talmor-Chohen A \& Shalgi R 2002 Signaling through protein kinases during egg activation. Journal of Reproductive Immunology 53 161-169.

Gallicano GI, McGaughey RW \& Capco DG 1992 Cytoskeletal sheets appear as universal components of mammalian eggs. Journal of Experimental Zoology 263 194-203.

Gallicano GI, McGaughey RW \& Capco DG 1995 Protein kinase M, the cytosolic counterpart of protein kinase $\mathrm{C}$, remodels the internal cytoskeleton of the mammalian egg during activation. Developmental Biology $167482-501$.

Gangeswaran R \& Jones KT 1997 Unique protein kinase C profiles in mouse oocytes: lack of calcium-dependent conventional isoforms suggested by RT-PCR and western blotting. FEBS Letters $\mathbf{4 1 2}$ 309-312.

Halet G, Tunwell R, Parkinson SJ \& Carroll J 2004 Conventional PKCs regulate the temporal pattern of $\mathrm{Ca}^{2+}$ oscillations at fertilization in mouse eggs. Journal of Cell Biology 164 1033-1044.

Inagaki M, Gonda $\mathbf{Y}$, Matsuyama M, Nishizawa K, Nishi Y \& Sato C 1987 Intermediate filament reconstitution in vitro. The role of phosphorylation on the assembly-disassembly of desmin. Journal of Biological Chemistry 263 5970-5978.

Johnson J \& Capco DG 1997 Progesterone acts through protein kinase $\mathrm{C}$ to remodel the cytoplasm, as the amphibian oocyte becomes fertilization-competent egg. Mechanisms of Development $67215-226$.

Jones KT 1998 Protein kinase C action at fertilization: overstated or undervalued? Reviews of Reproduction 3 7-12.

Kline D \& Kline JT 1992 Repetitive calcium transients and the role of calcium in exocytosis and cell cycle activation in the mouse egg. Developmental Biology 49 80-89.

Lelkes PI, Friedman JE, Rosenheck K \& Oplatka A 1986 Destabilization of actin filaments as a requirement for the secretion of catecholamines from permeabilized chromaffin cells. FEBS Letters $208357-363$.

Luria A, Tennenbaum T, Sun QY, Rubinstein S \& Breitbart H 2000 Differential localization of conventional protein kinase $\mathrm{C}$ isoforms during mouse oocyte development. Biology of Reproduction 62 1564-1570.

McAvery AB, Wortzman GB, Williams CJ \& Evans JP 2002 Involvement of calcium signaling and the actin cytoskeleton in the membrane block to polyspermy in mouse eggs. Biology of Reproduction 67 1342-1352.

Muallem S, Kwiatkowaska K, Xu X \& Yin HL 1995 Actin filament disassembly is a sufficient final trigger for exocytosis in nonexcitable cells. Journal of Cell Biology 128 589-598.

Nishizuka Y 1988 The molecular hetrogeneity of protein kinase C and its implications for cellular regulation. Nature 334 661-665.

Pauken CM \& Capco DG 2000 The expression and stagespecific localization of protein kinase $C$ isotypes during mouse preimplantation development. Developmental Biology 223 $411-421$.

Raz T \& Shalgi R 1998 Early events in mammalian egg activation. Human Reproduction 13 133-145.

Raz T, Ben-Yosef D \& Shalgi R 1998a Segregation of the pathways leading to the cortical reaction and cell cycle activation in the rat egg. Biology of Reproduction 58 94-102.

Raz T, Eliyahu E, Yesodi V \& Shalgi R 1998b Profile of protein kinase $\mathrm{C}$ isoenzymes and their possible role in mammalian egg activation. FEBS Letters 431 415-418.
Roeder AD \& Gard DL 1994 Confocal microscopy of F-actin distribution in Xenopus oocytes. Zygote 2 111-124.

Rosen A, Keenan KF, Thelen M, Nairn AC \& Aderem A 1990 Activation of protein kinase $C$ results in the displacement of its myristoylated, alanine-rich substrate from punctate structures in macrophage lopodia. Journal of Experimental Medicine $\mathbf{1 7 2}$ $1211-1215$.

Rossi EA, Li Z, Feng H \& Rubin CS 1999 Characterization of the targeting, binding, and phosphorylation site domains of an A kinase anchor protein and a myristoylated alanine-rich $C$ kinase substratelike analog that are encoded by a single gene. Journal of Biological Chemistry $27427201-27210$.

Runft LL, Jaffe LA \& Mehlmann LM 2002 Egg activation at fertilization: where it all begins. Developmental Biology 245 237-254.

Sha'afi RI \& Molski TF 1987 Signaling for increased cytoskeletal actin in neutrophils. Biochemical and Biophysical Research Communications 145 934-941.

Sontag JM, Aunis D \& Bader MF 1988 Peripheral actin filaments control calcium-mediated catecholamine release from streptolysin-opermeabilized chromaffin cells. European Journal of Cell Biology $46316-326$.

Spudich A, Wrenn JT \& Wessells NK 1988 Unfertilized sea urchin eggs contain a discrete shell of actin that is subdivided into two organizational states. Cell Motility and the Cytoskeleton 9 $85-96$.

Sun QY, Lai L, Park KW, Kuhholzer B, Prather RS \& Schatten H 2001 Dynamic events are differently mediated by microfilaments, microtubules, and mitogen-activated protein kinase during porcine oocyte maturation and fertilization in vitro. Biology of Reproduction 64 879-889.

Sutovsky P, Oko R, Hewitson L \& Schatten G 1997 The removal of the sperm perinuclear theca and its association with the bovine oocyte surface during fertilization. Developmental Biology $\mathbf{1 8 8}$ $75-84$.

Swann K \& Parrington J 1999 Mechanism of $\mathrm{Ca}^{2+}$ release at fertilization in mammals. Journal of Experimental Zoology 285 $267-275$.

Swierczynski SL \& Blackshear PJ 1995 Membrane association of the myristoylated alanine-rich C kinase substrate (MARCKS) protein. Mutational analysis provides evidence for complex interactions. Journal of Biological Chemistry 270 13436-13445.

Tahara M, Tasaka K, Masumoto N, Mammoto A, Ikebuchi Y \& Miyake A 1996 Dynamics of cortical granule exocytosis at fertilization in living mouse eggs. American Journal of Physiology $\mathbf{2 7 0}$ C1354-C1361.

Takashi S 1997 Reorganization of the cortical actin cytoskeleton during maturation division in the Tubifex egg: possible involvement of protein kinase C. Developmental Biology 188 110-121.

Talmor A, Kinsey WH \& Shalgi R 1998 Expression and immunolocalization of $\mathrm{p} 59^{\mathrm{c}-\mathrm{Fyn}}$ tyrosine kinase in rat eggs. Developmental Biology 194 38-46.

Tchakarov L, Zhang L, Rose SD, Tang R \& Trifaro JM 1998 Light and electron microscopic study of changes in the organization of the cortical actin cytoskeleton during chromaffin cell secretion. Journal of Histochemistry and Cytochemistry 46 193-203.

Terada Y, Simerly C \& Schatten G 2000 Microfilament stabilization by jasplakinolide arrests oocyte maturation, cortical granule exocytosis, sperm incorporation cone resorption, and cell-cycle progression, but not DNA replication, during fertilization in mice. Molecular Reproduction and Development 56 89-98.

Trifaro JM, Rodriguez Del Castillo A \& Vitale ML 1992 Dynamic changes in chromaffin cell cytoskeleton as a prelude to exocytosis. Molecular Neurobiology 6 339-358.

Trifaro JM, Lejen T, Rose SD, Dumitrescu Pene T, Barkar ND \& Seward EP 2002 Pathways that control cortical F-actin dynamics during secretion. Neurochemical Research 271 1371-1385.

Vitale ML, Rodriguez del Castillo A, Tchakarov L \& Trifaro JM 1991 Cortical filamentous actin disassembly and scinderin redistribution during chromaffin cell stimulation precede exocytosis: a 
phenomenon not exhibited by gelsolin. Journal of Cell Biology 113 1057-1067.

Wang WH, Abeydeera LR, Prather RS \& Day BN 2000 Polymerization of nonfilamentous actin into microfilaments is an important process for porcine oocyte maturation and early embryo development. Biology of Reproduction 62 1177-1183.

Welch MD, Mallavarapu A, Rosenblatt J \& Mitchison JJ 1997 Actin dynamics in vitro. Current Opinion in Cell Biology 9 54-61.
Wu WC, Walaas SI, Nairn AC \& Greengard P 1982 Calcium/ phospholipid regulates phosphorylation of a Mr " $87 \mathrm{k}^{\text {" substrate }}$ protein in brain synaptosomes. PNAS 79 5249-5253.

Received 12 July 2004

First decision 19 August 2004

Accepted 1 October 2004 